\title{
PID Neural Network Decoupling Control of Multi-variable System and its Application
}

\author{
DongJiang $\mathrm{Yu}^{1,}$, WeiZhi Long ${ }^{2}$, JiaBing $\mathrm{He}^{2}$ \\ ${ }^{1}$ Datang Power Generation Group Hunan Branch, Hunan, 410011, China \\ ${ }^{2}$ Datang Huayin Youxian Energy Corporation Limited, Luoyang, 412307, China \\ aemail:1653457448@qq.com
}

Keywords: MPIDNN; Decoupling Control; Multi-variable; Simulation

\begin{abstract}
In this thesis, multi-output PID neural network (MPIDNN) is proposed based on the research on single-output PID and it is simulated. MPIDNN is also proposed for the characteristics of coupling system which is difficult to control in industrial process. The results of the simulation show that the control algorithm has the function of online learning to adjust parameters. For multi-input and multi-output system, by using the MPIDNN decoupling control method, there is no need to get the exact model of object. It can offset the effects of the internal model or other disturbance and achieve better control effect.
\end{abstract}

\section{Introduction}

PID neural network (PIDNN) is a new neural network. It is a multilayer feedforward neural network composed of proportional (P), integral (I) and differential (D). PIDNN has the dual advantage of PID control and neural network control[1]. For multi-variable system which has serious coupling and time-varying, PID neural network uses the deviation of the system output and set value through online learning to modify the connection weights of the network to change strength of the role of proportional element, integration element and differential element. So as to achieve the purpose of decoupling control. PIDNN has a good dynamic performance and static performance, it has been used in many control systems and achieved good control effect[2-4].

A multi-output PID neural network control method is proposed for multivariable coupling objects and it is simulated based on the study of single variable PID neural network.

\section{The single-output PID neural network controller}

\subsection{Structure of single-output PID neural network controller}

Single-output PID neural network (Single-output PIDNN, SPID) is the basic form of PIDNN and its structure is three-tier. It has six neurons, each neuron consists of input of neurons, state of neurons and output of neurons[5]. The structure of controller is shown in figure 1.

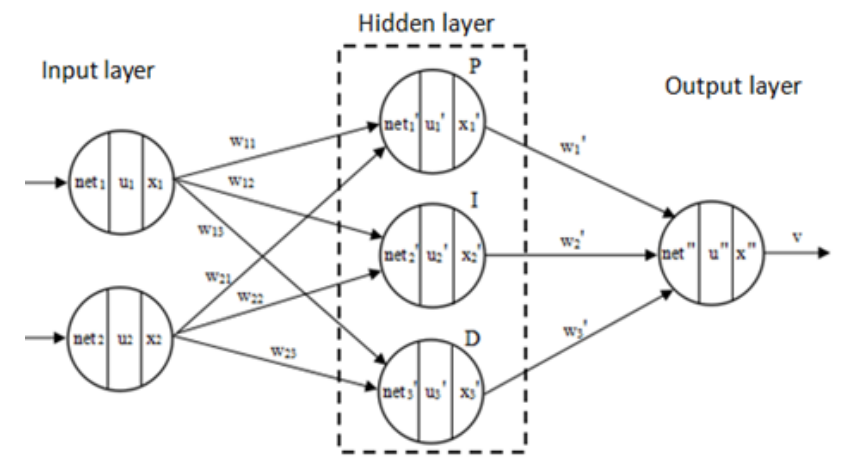

Fig.1. SPINN chart

The input layer of SPINN has two neurons: One receives a set output value of the controlled object with single-input and single-output, the other receives an actual output value of the controlled object with single-input and single-output. There are three neurons in hidden layer which 
are the proportional element (P) with proportional operation function, integral element (I) with operation function and differential element (D) with differential operation function. Output layer has only one neuron and its role is to synthesize and output[6].

2.2 SPINN forward algorithm of single-output PID neural network controller

Forward algorithm means the input value through the input layer, hidden layer and output layer sequentially.

(1) The input layer

The input layer contains two inputs of the set value $r$ and the actual output value $y$ respectively of controlled object of system. net represents the input of the input layer neurons, $u$ represents the state of the input layer neurons, $x$ represents the output neurons in the input layer and $i$ represents the sequence number of neurons in the input layer. At any sampling instant $k$, the input of the input layer neurons is

$$
\begin{aligned}
& \text { net }_{1}(k)=r(k) \\
& \text { net }_{2}(k)=y(k)
\end{aligned}
$$

(2) The hidden layer

Hidden layer is the core layer of SPIDNN, comprising three neurons. net' represents the input of the hidden layer neurons, $u^{\prime}$ represents the state of the hidden layer neurons, $x^{\prime}$ represents the output neurons of the hidden layer and $j$ represents the sequence number of hidden layer neurons.

$$
\operatorname{net}_{j}^{\prime}(k)=\sum_{i=1}^{2} w_{i j} x_{i}(k) \quad j=1,2,3
$$

(3) The output layer

It is responsible for collecting the output of each hidden layer neurons to achieve integrated output of the network. With net" represents the input of the output layer neurons, $u$ " represents the state of the output layer neurons, $x^{\prime \prime}$ represents the output neurons of the output layer, $j$ represents the sequence number of neurons of output layer.

$$
\operatorname{net}^{\prime \prime}(k)=\sum_{j=1}^{3} w_{j}^{\prime} x_{j}^{\prime}(k)
$$

$w_{j}^{\prime}$ on behalf of weights of the $j$-th neurons of hidden layer which connected to the output layer neurons.

The state of neuron in this layer is

$$
u^{\prime \prime}(k)=n e t^{\prime \prime}(k)
$$

The output of neuron in this layer is

$$
x^{\prime \prime}(k)=\left\{\begin{array}{cc}
1, & u^{\prime \prime}(k)>1 \\
u^{\prime \prime}(k), & -1 \leq u^{\prime \prime}(k) \leq 1 \\
-1, & u^{\prime \prime}(k)<-1
\end{array}\right.
$$

$v(k)$ is output of SPIDNN controller which is equal to the output of the output layer neurons.

$$
v(k)=x^{\prime \prime}(k)
$$

The algorithm modifies the network weights by objective function. The purpose of algorithm is to minimize the objective function.

$$
J=\frac{1}{m} \sum_{k=1}^{m}[r(k)-y(k)]^{2}=\frac{1}{m} \sum_{k=1}^{m} e^{2}(k)
$$

$m$ represents the sequence number of sampling points in each training session.

Weights of SPIDNN in each layer adjusted by the gradient method, learning step is $\eta$. After $n_{0}$-step training and learning, the corrected formula of weights in each layer is

$$
w\left(n_{0}+1\right)=w\left(n_{0}\right)-\eta \frac{\partial E}{\partial w}
$$

Weights of SPIDNN between the layers are adjusted based on the deviation of the output value of controlled object and the set value of controlled object. By online learning and self-tuning, 
SPIDNN does well without precise mathematical models and parameters and it can be applied to the control system with varying model parameters[7].

\section{Multi-output PID neural network controller}

3.1 Structure of multi-output PID neural network controller

Multi-output PID neural network (Multi-output PIDNN, MPIDNN) is combined into a plurality of SPIDNN. Each SPIDNN is a subnet of MPIDNN. MPIDNN is a three-forward network. MPIDNN requires the network connection of $n$-SPIDNN, if the controlled object has $m$ inputs and $n$ outputs. So this MPIDNN constitutes forward three networks whose structure is $2 n \times 3 n \times m$. The corresponding structure form of this MPIDNN control system is shown in figure 2.

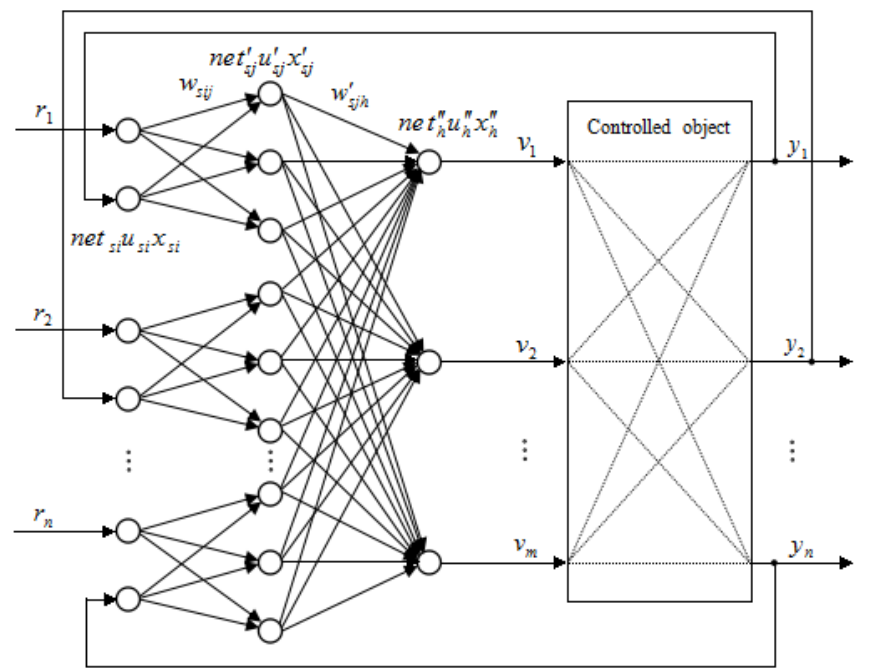

Fig.2. Architecture of MPIDNN control system with m inputs and n outputs

3.2 Algorithm of multi-output PID neural network controller

3.2.1 Forward algorithm of MPIDNN

Forward algorithm of MPIDNN is similar to forward algorithm of SPIDNN. Due to the controlled object has $m$ inputs, there are $m$ neurons in output layer of MPIDNN. $h \quad(h=1,2, \cdots, m)$ is the sequence number of neurons in the output layer. The input of output layer neuron is the sum of products of every output value of hidden layer neurons and the weight of the corresponding channel. Formula is as follows.

$$
\operatorname{net}_{h}^{\prime \prime}(k)=\sum_{s=1}^{n} \sum_{j=1}^{3} w_{s j h}^{\prime} x_{s j}^{\prime}(k)
$$

$w_{s j h}^{\prime}$ is the weight of the $j$-th neuron of the $s$-th hidden layer to the $h$-th neuron of output layer.

3.2.2 Back propagation algorithm of MPIDNN

The objective function is

$$
J=\sum_{p=1}^{n} E_{p}=\frac{1}{l} \sum_{p=1}^{n} \sum_{k=1}^{l}\left[r_{p}(k)-y_{p}(k)\right]^{2}
$$

$l$ represents the number of sampling points in training session, $n$ is the number of controlled variables, $p(p=1,2, \cdots, n)$ represents sequence of the output variable of controlled object.

(1) The hidden layer to the output layer

After training of $n_{0}$ steps, correction formula of weights of the $s$-th subnet in the hidden layer to the $h$-th neuron of output layer is as follows.

$$
w_{s j h}^{\prime}\left(n_{0}+1\right)=w_{s j h}^{\prime}\left(n_{0}\right)-\eta_{s j h}^{\prime} \frac{\partial J}{\partial w_{s j h}^{\prime}}
$$

Coupling relationship is between the output and the input of object with multiple variables. So there is the following formula. 


$$
\frac{\partial J}{\partial w_{s j h}^{\prime}}=\sum_{p=1}^{n} \frac{\partial J}{\partial E_{p}} \cdot \frac{\partial E_{p}}{\partial y_{p}} \cdot \frac{\partial y_{p}}{\partial v_{h}} \cdot \frac{\partial v_{h}}{\partial x_{h}^{\prime \prime}} \cdot \frac{\partial x_{h}^{\prime \prime}}{\partial u_{h}^{\prime \prime}} \cdot \frac{\partial u_{h}^{\prime \prime}}{\partial n e t_{h}^{\prime \prime}} \cdot \frac{\partial n e t_{h}^{\prime \prime}}{\partial w_{s j h}^{\prime}}
$$

It can be derived as follows.

$$
\frac{\partial J}{\partial w_{s j h}^{\prime}}=-\frac{2}{l} \sum_{p=1}^{n} \sum_{k=1}^{l}\left[r_{p}(k)-y_{p}(k)\right] \cdot \operatorname{sgn}\left[\frac{y_{p}(k+1)-y_{p}(k)}{v_{h}(k)-v_{h}(k-1)}\right] \cdot x_{s j}^{\prime}(k)
$$

There is a hypothesis.

$$
\delta_{h p}^{\prime}(k)=2\left[r_{p}(k)-y_{p}(k)\right] \cdot \operatorname{sgn}\left[\frac{y_{p}(k+1)-y_{p}(k)}{v_{h}(k)-v_{h}(k-1)}\right]
$$

Correction formula of weights of the $j$-th neuron of the $s$-th subnet in the hidden layer to the $h$-th neuron of output layer is as follows.

$$
w_{s j h}^{\prime}\left(n_{0}+1\right)=w_{s j h}^{\prime}\left(n_{0}\right)+\eta_{s j h}^{\prime} \cdot \frac{1}{l} \sum_{p=1}^{n} \sum_{k=1}^{l} \delta_{h p}^{\prime}(k) \cdot x_{s j}^{\prime}(k)
$$

(2)The input layer to the hidden layer

Correction formula of weights of the $i$-th neuron of the $s$-th subnet in the input layer to the $j$-th neuron of hidden layer is as follows.

$$
w_{s i j}\left(n_{0}+1\right)=w_{s i j}\left(n_{0}\right)-\eta_{s i j} \frac{\partial J}{\partial w_{s i j}}
$$

$i(i=1,2)$ is the sequence number of neurons in input layer of the subnet.

\section{Examples of simulation}

In order to verify online learning decoupling control of PIDNN, we used an example of two inputs and two outputs in time-varying system. For testing the robustness of the controller, the thesis assumes the transfer function of the controlled object becomes $G_{2}$ after 200 seconds. In order to test the actual response of the controlled object before its parameters changed and after its parameters changed, the thesis assumes that the fixed input signal value is $V$.

$$
\begin{aligned}
& G_{1}=\left[\begin{array}{ll}
G_{11}(s) & G_{21}(s) \\
G_{12}(s) & G_{22}(s)
\end{array}\right]=\left[\begin{array}{cc}
\frac{1.298}{7.852 s+1} & \frac{0.182}{8.768 s+1} \\
\frac{0.214}{6.584 s+1} & \frac{0.852}{9.231 s+1}
\end{array}\right] \\
& V=\left[\begin{array}{c}
v_{1}(t) \\
v_{2}(t)
\end{array}\right]=\left[\begin{array}{c}
0.8(t-5)-0.4(t-120) \\
0.3(t-5)+0.7(t-60)
\end{array}\right]
\end{aligned}
$$

The corresponding output curve of open-loop is shown in figure 3.
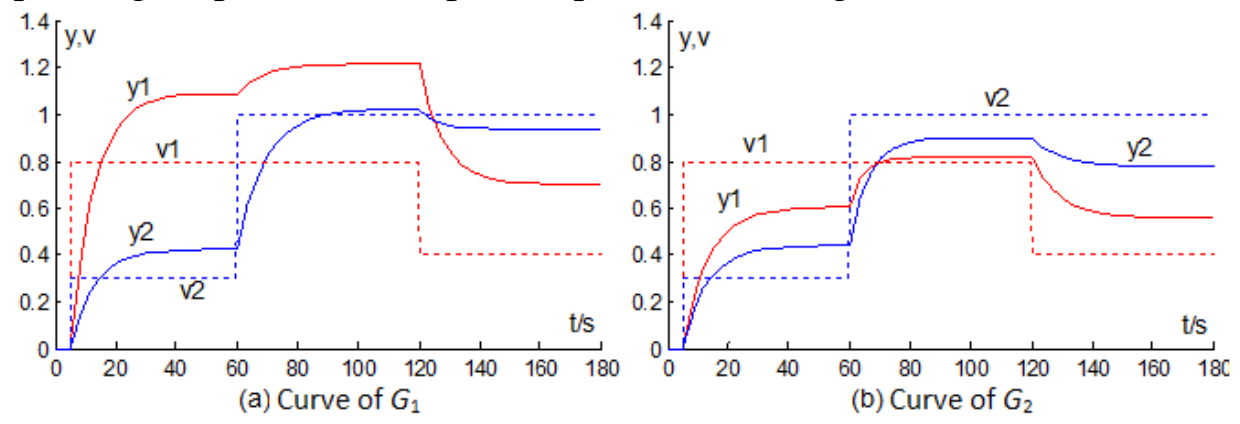

Fig.3. The response curve of open-loop with unchanged-parameters and changed-parameters of controlled object of the two inputs and two outputs

The response curve of controlled object is different with the same input. This is because the object's parameters are changed. Two outputs of controlled object will be affected if one input changed and the other input unchanged. This means that there is a strong coupling between the 
controlled object.

\subsection{Control of MPIDNN}

The structure of the object with two inputs and two outputs is shown in figure 4 . The structure of the control system is $4 \times 6 \times 2$.

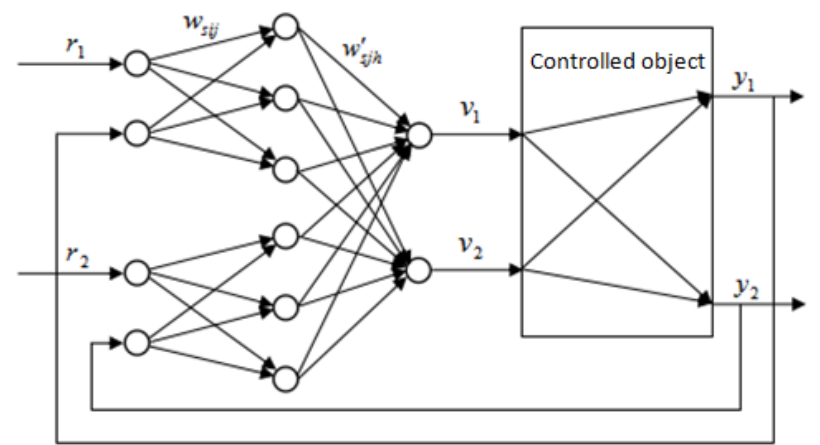

Fig.4. Structure of the MPIDNN control system of the object with two inputs and two outputs

The initial value of weights of the input layer to the hidden layer is $w_{s 11}(0)=w_{s 12}(0)=w_{s 13}(0)=1, w_{s 21}(0)=w_{s 22}(0)=w_{s 23}(0)=-1, s$ representatives sequence number of subnets. $s=1,2$. The initial value of weights of the hidden layer to the output layer is set in accordance with PID parameters which are suitable to $G_{1}$. The initial value of weights of three neurons of the hidden layer to the first neuron of the output layer is correspond to PID1 parameters respectively, the initial value of weights of three neurons of the hidden layer to the second neuron of the output layer is 0 in the first subnet. The initial value of weights of three neurons of the hidden layer to the second neuron of the output layer is correspond to PID2 parameters respectively, the initial value of weights of three neurons of the hidden layer to the first neuron of the output layer is 0 in the second subnet. PID parameters are selected with particle swarm optimization. Ignore internal coupling between the controlled object. PID1 parameters are selected according to the transfer function of $G_{11}$. $G_{11}$ is from $v_{1}$ to $y_{1}$. PID2 parameters are selected according to the transfer function of $G_{22}$. $G_{22}$ is from $v_{2}$ to $y_{2}$. PID parameters which obtained through optimization when the transfer function of the controlled object is $G_{1}$ are as follows. $k_{p 1}=5.663, k_{i 1}=0.769, k_{p 2}=10.246, k_{i 2}=1.173$. When the transfer function of the controlled object is $G_{2}$, PID parameters are as follows. $k_{p 1}^{\prime}=12.723, k_{i 1}^{\prime}=1.540, k_{p 2}^{\prime}=7.782, k_{i 2}^{\prime}=1.522$.

Experimental procedure of MPIDNN is as follows.

(1) If $t$ is 0 and the controlled object is $G_{1}$, optimization parameters used by the PID controller corresponds with the $G_{1}$;

(2) If $t$ is 200 seconds and the controlled object is $G_{2}$, optimization parameters used by the PID controller corresponds with the $G_{2}$;

(3) If $t$ is 300 seconds, rejoined the disturbance of a given value. Compare MPIDNN control and traditional PID control. The simulation results is shown in figure 5.
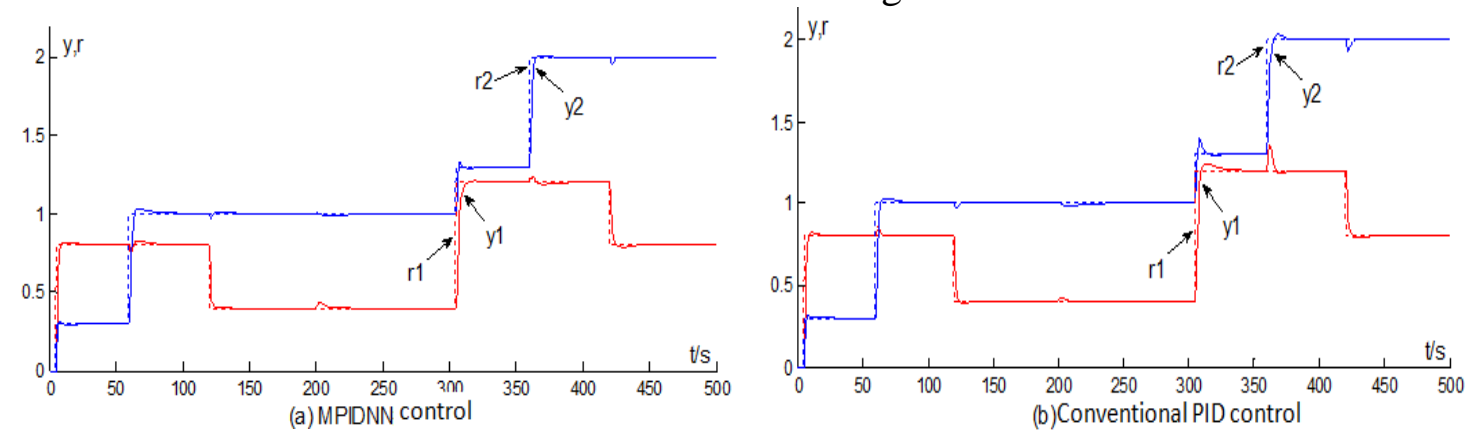

Fig.5. Comparison chart of MPIDNN control and traditional PID control performance

As can be seen, MPIDNN has a similar effect with traditional PID because of MPIDNN inherited traditional PID parameters when the beginning of the experiment. With weights of 
MPIDNN learned and updated continuously, MPIDNN has had the ability to decouple. And the influences of the internal disturbance on output of MPIDNN is smaller than influences of traditional PID control. After 200 seconds, controlled object has changed and traditional PID parameters have updated. But traditional PID still not achieve a good control effect for internal coupling, internal disturbance overshoot is large. Due to MPIDNN has the function of online learning to adjust network weights in the control process, MPIDNN not only adapts to a new controlled object and offsets the disturbance caused by the internal coupling. When the controlled object is changed, MPIDNN still able to maintain good control of the decoupling, small overshoot and settling time is short. MPIDNN has a stronger ability to adapt than traditional PID.

\section{Conclusion}

If the parameters of the system object are unknown, PIDNN controller can achieve the effect of decoupling control through its own study and training. Compared to traditional PID controller, advantages of PIDNN controller are fast response, small overshoot, settling time is short, adaptive and anti-interference ability is strong, etc. PIDNN controller can achieve a good effect of decoupling control for the system with serious coupling and time-varying and parameters of internal model are unknown.

\section{References}

[1]Guo Aiwen, Li Jinping, Yang Jiebin. PIDNN Decoupling Control of Doubly Fed Hydro-generator System Based on PSO Method[C]. Control Conference (CCC), 2011 30th Chinese. IEEE, 2011: 2698-2701

[2]Liu Changhua, Song Hua. Design of Electric Loading System in Flight Simulator Based on PIDNN[C]. Mechatronic Science, Electric Engineering and Computer (MEC), 2011 International Conference on. IEEE, 2011: 2623-2626

[3]Weijie Dong, Xiaomin Bai, Ninghui Zhu, et al. Active Power Filter Based on PIDNN Controller[C]. Innovative Smart Grid Technologies-Asia (ISGT Asia), 2012 IEEE. IEEE, 2012: 1-4

[4]Lixiong Lin, Xiafu Peng. A PID Neural Network Control for Permanent Magnet Synchronous Motor Servo System[C]. Computer Science and Education (ICCSE), 2010 5th International Conference on. IEEE, 2010: 1174-1178

[5]Hua Shu, Yuan-kun Xu. Application of Additional Momentum in PID Neural Network[C]. Information Science and Technology (ICIST), 2014 4th IEEE International Conference on. IEEE, 2014: 172-175

[6]Elamvazuthi, I., Aymen, O.M., Salih, Y., et al. An Intelligent Control of Blood Pressure System Using PID and Neural Network[C]. Industrial Electronics and Applications (ICIEA), 2013 8th IEEE Conference on. IEEE, 2013: 1049-1053

[7]Faa-Jeng Lin, Jonq-Chin Hwang, Kuang-Hsiung Tan, et al. Control of Doubly-Fed Induction Generator System Using PIDNNs[C]. Machine Learning and Applications (ICMLA), 2010 Ninth International Conference on. IEEE, 2010: 675-680 